$85^{\text {th }}$ and ro5th, and finally that between the $15^{\text {th }}$ and 14 oth degrees. But the intensity of colour must vary inversely to the breadth of the stripes, and the three stripes left between the red ones be filled with a pretty vivid blue. This hemisphere placed upon a table with its southern pole pointing towards sunset will atford a tolerable portrait of the aspect of the sky as it appeared immediately after sunset, and continued unchanged for more than a quarter of an hour. The stripes were not visible near the horizon, but were very distinct at an altitude of about fifteen degrees, and almost disappeared about the zenith. No cloud was seen during the occurrence of the phenomenon.

These stripes were certainly parallel in reality, and their apparent divergence may be accounted for by perspective. The redidish stripes may owe their colour to sunlight reflected back from the particles scattered in the atmosphere. But why did the celestial vault show so distinct a blue colour in the intervening bands? Yet, probably, this phenomenon is more easily to be explained than the infinite variation of evening colourings that want a valid explanation to this day.

Magdeburg, August 19

A. SPRUNG

\section{THE AURORA}

THERE was a very fine display of aurora here on the night of the 2 ist. It commenced to be visible about 9.30 P.M., reached its maximum about II, and faded suddenly away about II.3o. In appearance it was of a silvery white, without a trace of that rose colour which characterised the three great displays of last autumu. The main portion of the light was in the north-western quarter of the heavens, and it was sufficiently strong to see large print by. Extending from the north-west and reaching the north-eastern horizon arose three luminous arches concentric with each other, the Ist about $15^{\circ}$ altitude, the 2 nd about $25^{\circ}$ altitude, and the 3 rd about $40^{\circ}$ altitude. These were connected by radial tongues of light which were ever changing their height. There was another marked and isolated nucleus about and around a Lyræ.

At about 10.45 P.M. there were most curious rays shot up from the arches in the north, and concentric with them These shooting arches, if I may call them so, had at the horizon an apparent angle of about $150^{\circ}$ to $180^{\circ}$, but as they approached the nucleus in Lyra, they contracted and lost themselves in sheets of white light. On applying the spectroscope I found one bright line visible all over the heavens excepting on the south horizon for an altitude of about $25^{\circ}$. The spectrum obtained on the north-west gave five bright lines, of which I send a drawing.

From want of convenient measuring apparatus I had resource to the method of superposed spectra. The light I chose for comparison was that of a tallow candle, from which I got the bright lines of sodium and carburetted

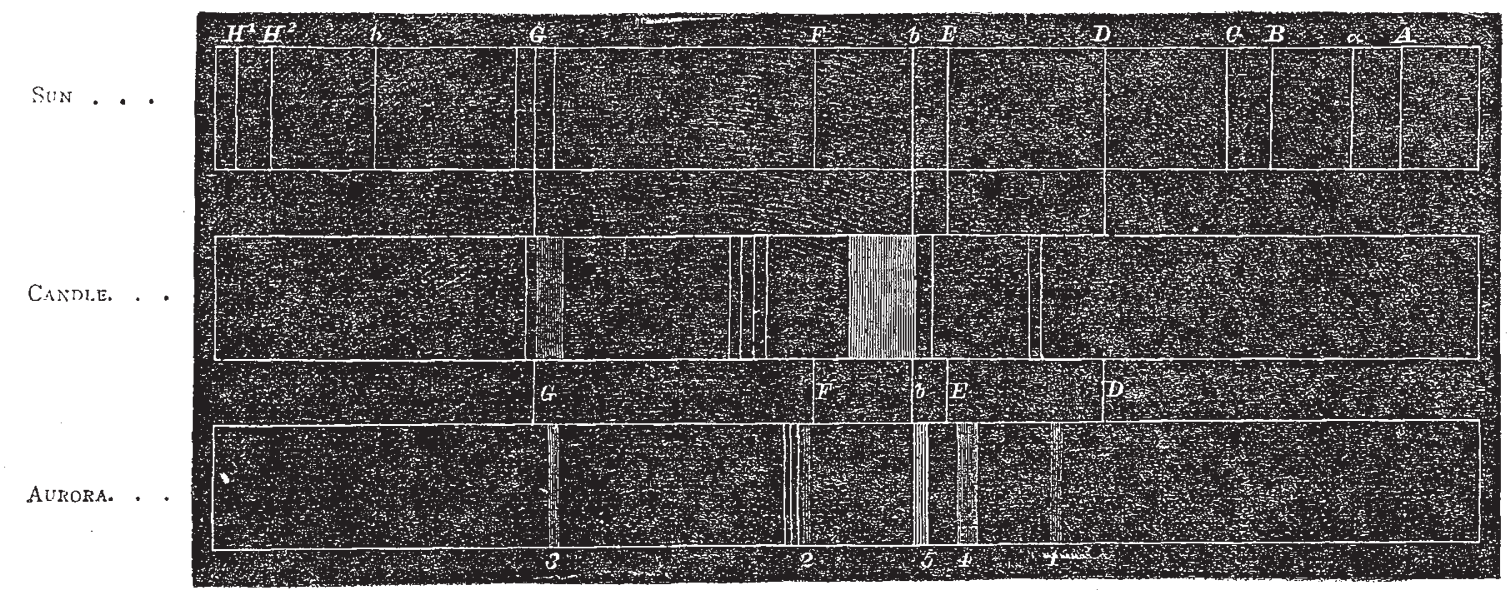

COMPARISON SPECTRA OF SUN, AURORA, AND CANDLE

hydrogen. 'The instrument I used was one of Browning's direct vision spectroscopes-an instrument that gives the best results with the minimum amount of light. Of the bright lines, two were strong, one was medium, two were very faint. In the accompanying map I have put the solar spectrum at the top and carried the chief lines down for comparison. In putting numbers to the lines I have been directed by their degrees of intensity.

No. I is a sharp, well-formed line, visible with a very narrow slit.

No. 2. A line very slightly more refrangible than $F$. The side towards D is sharp and well-defined, while on the other side it is nebulous.

No. 3. Slightly less refrangible than $G$, is a broad illdefined band only seen with a wide slit.

No. 4. A line near E, woolly at the edges, but rather sharp in the centre. This should be at or near the position of the line 1474 of the solar corona.

No. 5. A faint band coincident with $b$, extending equally on both sides of it.

The barometer stood at 29.574 in. ; the thermometer at $61^{\circ} 3$. A gentle wind was blowing from the south-west, and the sky was free from clouds.

Observatory, Dun Echt, Aberdeen

\section{FRUIT CLASSIFICATION *}

DR. DICKSON referred to the confessedly unsatisfactory state of fruit-classification, and to the very unnecessary extent of the existing terminology, which is further complicated by a considerable amount of variance among botanists as to the pre. cise application of several of the terms employed. He was of the opinion, which he believed to be a growing one among botanists, that the most convenient method of classification was, in the first place, rigorously to restrict the definition of a "fruit" to the mature or ripe pistil, excluding from that definition the modifications of accessory parts or organs, which, in many cases, are correlative therewith ; and, secondly, to base the primary classification upon the general character of the modification undergone by the parts of the pistil in ripening, treating as of minor importance the characters involved in the description of the flower, such as the superior or inferior position of the ovary, \&c.

The classification which Dr. Dickson suggests for the consideration of botanists approaches most nearly to that indicated by Schacht in his "Grundriss," of which, indeed, it may be viewed as a modification and expansion. Schacht grouped fruits under three heads-(I) Capsular fruits which dehisce to allow the seeds to escape ; (2) splitting fruits or Schizocarps, which

" "Suggestions on Fruit Classification." By Alex. Dickson, M.D.; Regius Professor of Botany in the University of Glasgow. Read lefore the Regius Professor of Botany
British Association, $187 \mathrm{r}$. 